\title{
114
}

[The following paper was read 8th March 1907.]

\section{On Vandermonde's Theorem, and some more general Expangions.}

\author{
By John Dougall, M.A.
}

1. If we write

$$
\left(\begin{array}{l}
a \\
8
\end{array}\right) \equiv \frac{a(a-1) \ldots(a-s+1)}{8 !},
$$

where $a$ is arbitrary, but $s$ is a positive integer, then Vandermonde's Theorem is

$$
\left(\begin{array}{c}
p+q \\
s
\end{array}\right)=\left(\begin{array}{c}
p \\
s
\end{array}\right)+\left(\begin{array}{c}
p \\
s-1
\end{array}\right)\left(\begin{array}{l}
q \\
1
\end{array}\right)+\left(\begin{array}{c}
p \\
s-2
\end{array}\right)\left(\begin{array}{l}
q \\
2
\end{array}\right)+\ldots \ldots+\left(\begin{array}{l}
q \\
s
\end{array}\right)
$$

Divide by $\left(\begin{array}{l}p \\ s\end{array}\right)$, and put $a$ for $q, s+\gamma$ for $p$.

The theorem takes the form

$\frac{(a+\gamma+1)(a+\gamma+2) \ldots(a+\gamma+s)}{(\gamma+1)(\gamma+2) \ldots(\gamma+s)}=1+\frac{a s}{1 \cdot \gamma+1}+\frac{a \cdot a-1 \cdot s \cdot s-1}{1 \cdot 2 \cdot \gamma+1 \cdot \gamma+2}+\ldots(1)$.

The formula may be regarded either as summing the series on the right, or as expanding, in a particular form, the function on the left. It is by proceeding from the latter point of view that the following developments suggest themselves.

As is well known, Vandermonde's Theorem is susceptible of a great variety of proofs. For instance, in either of the forms above, it is one of the simplest examples of Newton's Interpolation formula. The proof to be given here combines in a somewhat peculiar way the principles of symmetry and algebraic degree with the step by step method, or method of mathematical induction.

The idea of this method obviously admitting of extension, other functions are invented, capable of expansion in finite series of factorials of suitable form. By increasing the number of terms indefinitely, various interesting infinite series are summed in terms of $I I$ functions, in particular the hypergeometric series with fourth element unity, and several series involving the third and fourth powers of the coefficients in the expansion of $(1-x)^{-0}$. 
Although hardly within the scope of the paper, one or two examples are added of a method of extending some of these summation theorems with the help of Cauchy's Theory of Residues.

2. The proof we propose to give of the theorem (1) depends mainly on this, that when $a$, like $s$, is a positive integer, the fraction on the left is symmetrical in $a$ and $s$. In fact, when we multiply both numerator and denominator by $(\gamma+1)(\gamma+2) \ldots(\gamma+\alpha)$, the fraction becomes

$$
\frac{(\gamma+1)(\gamma+2) \ldots(\gamma+a+s)}{(\gamma+1) \ldots(\gamma+\alpha) \times(\gamma+1) \ldots(\gamma+s)}
$$

the symmetry of which is obvious.

When $a$ is zero, the fraction is unity. Now we shall define the value of a factorial $(x+1)(x+2) \ldots(x+s)$ to be unity when $s$ is zero, and the symmetry spoken of therefore extends even to zero values of $a$ and 8 .

The theorem is then true when $s=0$. Assume it true (for every $a$ ) when $s=0,1,2, \ldots(n-1)$. We shall prove it true for $s=n$.

For, when $s=n$, the theorem is true for $a=0,1,2, \ldots(n-1)$, everything being symmetrical in $s$, $a$, and the theorem being true by hypothesis for $a=n$ and $s=0,1,2, \ldots(n-1)$.

Also when $8=n$, the coefficient of the highest power of $\alpha$ is the same on both sides. We have therefore two rational integral functions of $a$ of degree $n$, equal for $n$ values of $a$ and with equal coefficients of $a^{n}$. The functions are therefore identically equal; which proves the theorem.

3. The factorial function

$$
\frac{(\alpha+\gamma+1) \ldots(\alpha+\gamma+s)}{(\gamma+1) \ldots(\gamma+s)} \cdot \frac{(\beta+\gamma+1) \ldots(\beta+\gamma+s)}{(\alpha+\beta+\gamma+1) \ldots(\alpha+\beta+\gamma+s)}
$$

is the quotient of the function on the left of (1) by a function of the same form with $\beta+\gamma$ for $\gamma$. The same symmetry in $s, a$ is therefore present. We shall show that the function may be expanded in the form

$$
1+\mathrm{A}_{1} \frac{a s}{a+\beta+\gamma+s}+\mathrm{A}_{2} \frac{a \cdot a-1 \cdot s \cdot s-1}{a+\beta+\gamma+s \cdot a+\beta+\gamma+s-1}+\ldots,
$$

with the coefficients $A_{1}, A_{2}, \ldots, A_{n}, \ldots$ independent of $s$ and $\alpha$. In the first place, if this be provisionally assumed, these coefficients can be found at once by putting $s=n$, multiplying by 
$(\alpha+\beta+\gamma+1) \ldots(\alpha+\beta+\gamma+n)$, and then putting $\alpha=-\beta-\gamma-1$.

Every term on the right disappears but the last, and we get

$$
\frac{\beta(\beta-1) \ldots(\beta-n+1)}{(\gamma+1) \ldots(\gamma+n)}=\mathrm{A}_{n} \cdot n ! .
$$

The theorem to be proved is then

$$
\begin{aligned}
& \frac{+\gamma+1) \ldots(a+\gamma+s)}{(\gamma+1) \ldots(\gamma+s)} \cdot \frac{(\beta+\gamma+1) \ldots(\beta+\gamma+s)}{(\alpha+\beta+\gamma+1) \ldots(a+\beta+\gamma+s)} \\
& =1+\frac{a \cdot \beta}{1 \cdot \gamma+1} \cdot \frac{s}{a+\beta+\gamma+s}+\frac{a \cdot a-1 \cdot \beta \cdot \beta-1}{1 \cdot 2 \cdot \gamma+1 \cdot \gamma+2} \cdot \frac{8 \cdot \beta-1}{a+\beta+\gamma+s \cdot a+\beta+\gamma+s-1}+. .
\end{aligned}
$$

For proof, assume it true when $8=0,1,2, \ldots(n-1)$. Then when $s=n$, we have, after multiplication by $(a+\beta+\gamma+1) . .(a+\beta+\gamma+n)$, two rational integral functions of $\alpha$ of degree $n$ equal for the $n$ values $0,1,2, \ldots(n-1)$, and the additional value $-\beta-\gamma-1$. These functions are therefore identical, and the theorem, being true for $s=0$, is trae for $s$ any positive integer.

4. The theorem (2) is noteworthy for two reasons. The chief is that it contains as a limiting case the highly important summation of the hypergeometric series with fourth element unity. To obtain this, take the limit of the two members of (2) for 8 infinite.

The left hand member may be written

$$
\begin{aligned}
& \frac{(\alpha+\gamma+1) \ldots(a+\gamma+s)}{s !} s^{-a-\gamma} \cdot \frac{s !}{(\gamma+1) \ldots(\gamma+s)} s^{\gamma} . \\
& \frac{(\beta+\gamma+1) \ldots(\beta+\gamma+s)}{s !} s-\beta-\gamma \cdot \frac{s !}{(\alpha+\beta+\gamma+1) \ldots(a+\beta+\gamma+s)} s^{a+\beta+\gamma} .
\end{aligned}
$$

But according to Gauss's definition of the $\Pi$ function,

$$
\Pi z=\operatorname{Limit}_{s=\infty} \frac{s !}{(z+1)(z+2) \ldots(z+s)} \varepsilon^{2}
$$

so that the limit of the left hand member of (2) is

$$
\frac{\Pi \gamma \cdot \Pi(\alpha+\beta+\gamma)}{\Pi(a+\gamma) \Pi(\beta+\gamma)} \text {. }
$$

(It may be noted here, in connection with the $\Pi$ function that the definition gives at once

$$
(z+1)(z+2) \ldots(z+n)=\frac{\Pi(n+z)}{\Pi z},
$$




\section{7}

so that the definition itself may be read

$$
\begin{aligned}
\operatorname{Lt}_{s=\infty} \frac{\Pi s}{\Pi(s+z)} s^{z} & =1, \\
\text { or } \quad(z+1)(z+2) \ldots(z+8) & \left.=\frac{1}{\Pi z} s^{2} \Pi s, \text { asymptotically. }\right)
\end{aligned}
$$

Hence the limit of the series on the right of (2) has this value, whatever be the relative values of $\alpha, \beta, \gamma$; this limit we can easily show to be equal to the series of limits of the individual terms in order, provided the real part of $\alpha+\beta+\gamma$ is not negative.

For under this restriction the moduli of the factors

$$
\frac{s}{a+\beta+\gamma+s}, \frac{s-1}{a+\beta+\gamma+s-1}, \ldots
$$

cannot exceed unity, whatever be the value of $s$. Hence the residue after $n$ terms of the series of moduli of the terms of (2) cannot exceed the corresponding residue in the absolutely converging series

$$
1+\frac{a \cdot \beta}{1 \cdot \gamma+1}+\frac{a \cdot a-1 \cdot \beta \cdot \beta-1}{1 \cdot 2 \cdot \gamma+1 \cdot \gamma+2}+\ldots .
$$

But we can choose $n$, independent of $s$, so that the latter residue is as small as we please, and, having so chosen $n$, we can then make $s$ infinite.

\section{Hence}

$$
1+\frac{a \cdot \beta}{1 \cdot \gamma+1}+\frac{a \cdot a-1 \cdot \beta \cdot \beta-1}{1 \cdot 2 \cdot \gamma+1 \cdot \gamma+2}+\ldots=\frac{\Pi \gamma \cdot \Pi(\alpha+\beta+\gamma)}{\Pi(\alpha+\gamma) \overline{\Pi(\beta+\gamma)}} .
$$

The equation (3) which we have proved subject to the restriction $\mathbf{R}(\alpha+\beta+\gamma) \nless 0$, holds, in fact, so long as the series converges, that is, so long as $R(\alpha+\beta+\gamma)>-1$.

The equation is obviously equivalent to the more familiar form $F(a, \beta, \gamma, 1) \equiv$

$$
1+\frac{a \cdot \beta}{1 \cdot \gamma}+\frac{\alpha \cdot a+1 \cdot \beta \cdot \beta+1}{1 \cdot 2 \cdot \gamma \cdot \gamma+1}+\ldots=\frac{\Pi(\gamma-1) \Pi(\gamma-\alpha-\beta-1)}{\Pi(\gamma-a-1) \Pi(\gamma-\beta-1)} .
$$

Vandermonde's Theorem is the special case of (3) for one of $a, \beta$ a positive integer.

5. A second point of interest about the equation (2) is that with its aid a proof by actual multiplication can be given of the theorem

$$
\mathrm{F}(\alpha, \beta, \gamma, x)=(1-x)^{\gamma-a-\beta} \mathrm{F}(\gamma-a, \gamma-\beta, \gamma, x) \text {. }
$$


For if we multiply

by

$$
\mathrm{F}(\alpha, \beta, \gamma, x)=1+\frac{\alpha \cdot \beta}{1 \cdot \gamma} x+\frac{\alpha \cdot \alpha+1 \cdot \beta \cdot \beta+1}{1 \cdot 2 \cdot \gamma \cdot \gamma+1} x^{2}+\ldots
$$

$$
(1-x)^{-(\gamma-a-\beta)}=1+\frac{\gamma-a-\beta}{1} x+\frac{\gamma-\alpha-\beta \cdot \gamma-\alpha-\beta+1}{1.2} x^{2}+\ldots
$$

and arrange the product by powers of $x$, as we are entitled to do, if $|x|<1$, the coefficient of $x^{n}$ is

$$
\begin{aligned}
& \frac{(\gamma-a-\beta) . .(\gamma-a-\beta+n-1)}{n !} \\
& \left\{1+\frac{a \cdot \beta}{1 \cdot \gamma} \frac{n}{\gamma-a-\beta+n-1}+\frac{a \cdot a+1 \cdot \beta \cdot \beta+1 \cdot n \cdot n-1}{1 \cdot 2 \cdot \gamma \cdot \gamma+1 \cdot \gamma-a-\beta+n-1 \cdot \gamma-\alpha-\beta+n-2}+\ldots\right. \\
& =\frac{(\gamma-a-\beta) . .(\gamma-a-\beta+n-1)}{n !} \cdot \frac{(\gamma-a) . .(\gamma-a+n-1)}{\gamma \ldots(\gamma+n-1)} \cdot \frac{(\gamma-\beta) \ldots(\gamma-\beta+n-1)}{(\gamma-\alpha-\beta) \cdot .(\gamma-\alpha-\beta+n-1}
\end{aligned}
$$

as we find from (2) by changing $a$ into $-\alpha, \beta$ into $-\beta, \gamma$ into $\gamma-1$, and $s$ into $n$.

This is the same as the coefficient of $x^{n}$ in $\mathrm{F}(\gamma-\alpha, \gamma-\beta, \gamma, x)$, whence the theorem.

It is also worthy of remark that just as (2) has been used to prove (4), so Vandermonde's Theorem (1) may be used to prove the equally important relation

$$
\mathrm{F}(\alpha, \beta, \gamma, x)=(1-x)^{-\alpha} \mathrm{F}\left(\alpha, \gamma-\beta, \gamma, \frac{x}{x-1}\right) .
$$

Having found (5) in this way, we might deduce (4) in either of two ways; (i), by applying to $\mathrm{F}\left(\gamma-\beta, a, \gamma, \frac{x}{x-1}\right)$ the same transformation as is applied to $\mathrm{F}(\alpha, \beta, \gamma, x)$ in (5), thus finding

$$
\mathrm{F}\left(\alpha, \gamma-\beta, \gamma, \frac{x}{x-1}\right)=\left(1-\frac{x}{x-1}\right)^{\beta-\gamma} \mathrm{F}(\gamma-\beta, \gamma-\alpha, \gamma, x) ;
$$

or (ii), simply from the symmetry of $\mathrm{F}(\alpha, \beta, \gamma, x)$ in $\alpha, \beta$ by deriving from (5)

$$
(1-x)^{-\alpha} \mathrm{F}\left(\alpha, \gamma-\beta, \gamma, \frac{x}{x-1}\right)=(1-x)^{-\beta} \mathrm{F}\left(\beta, \gamma-\alpha, \gamma, \frac{x}{x-1}\right),
$$

which is merely another way of writing (4).

By using the latter method, it will be seen that through the medium of these hypergeometric series we might derive (2) from (1). 
6. In proving (1) and (2) we have taken advantage of two properties of such a factorial function as $\frac{(x+a+1) \ldots(x+a+8)}{(a+1) \ldots(a+) 8}$, namely, that it is of degree 8 in $x$, and that when $x$, like $s$, is a positive integer, it is symmetrical in $s, x$. By joining to these a third property of factorials we can invent a much more general expansion.

The new property is that the product of two factorials

$$
(x+a+1) \ldots(x+a+8) \text { and }(x+b+1) \ldots(x+b+8)
$$

is not altered by the substitution of $-x-a-b-s-1$ for $x$.

In fact obviously $(x+p)(x+q)$ remains unchanged when $-x-p-q$ is written for $x$, and the factors of the above product can be arranged in pairs

$$
(x+a+1)(x+b+s),(x+a+2)(x+a+b+s-1), \ldots
$$

of the form $(x+p)(x+q)$, with $p+q$ the same for all, namely, equal to $a+b+s+1$.

Consider now the function

$$
\frac{(x+a+1) \ldots(x+a+s) \cdot(x+b+1) \ldots(x+b+s)}{x+c+1) \ldots(x+c+s) \cdot(x+d+1) \ldots(x+d+8)} \cdot \frac{(c+1) \ldots(c+s) \cdot(d+1) \ldots(d+s)}{(a+1) \ldots(a+s) \cdot(b+1) \ldots(b+s)} \text {. }
$$

This has clearly the property of symmetry in $x, s$ when $x$ is a positive integer. It will also have the property of remaining unchanged when $-x-a-b-s-1$ is put for $x$, provided $a+b=c+d$.

It can be expanded in a series of functions of $x, s$, all of which possess these two properties, namely, in the form

$$
\begin{aligned}
1 & +\mathrm{A}_{1} \frac{x \cdot s \cdot x+a+b+s+1}{x+c+1 \cdot s+c+1 \cdot x+d+s} \\
& +\mathrm{A}_{2} \frac{x \cdot x-1 \cdot s \cdot s-1 \cdot x+a+b+s+1 \cdot x+a+b+s+2}{x+c+1 \cdot x+c+2 \cdot s+c+1 \cdot s+c+2 \cdot x+d+s \cdot x+d+8-1}+\ldots ;
\end{aligned}
$$

where the A's are independent of $s$ and $x$.

The proof is practically the same as in $\S 3$. Thus, assuming the theorem for a moment, we determine $A_{n}$ by giving $s$ the value $n$, multiplying by

$$
(x+c+1) \ldots(x+c+n) \cdot(x+d+n) \ldots(x+d+1),
$$

and putting $-d-1$ (or $-c-n)$ for $x$. 
\&

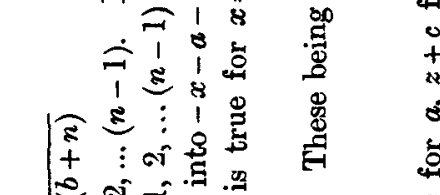

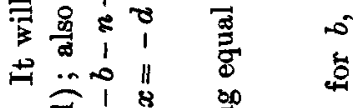

दิ

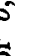

+.

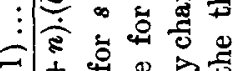

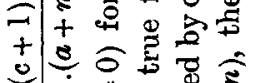

落

1)

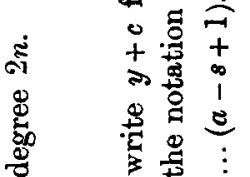

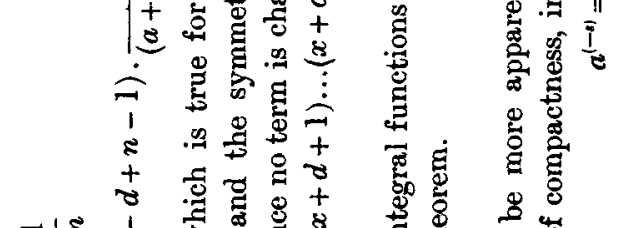

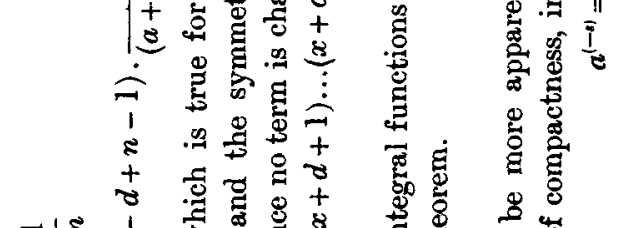

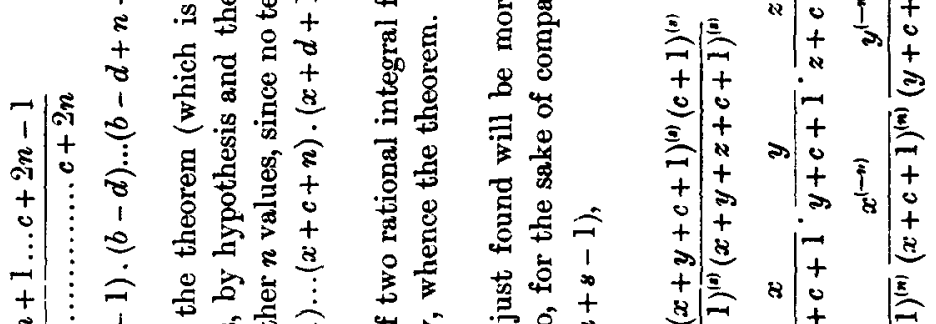

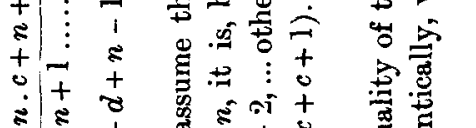

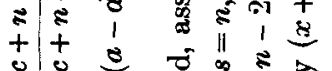

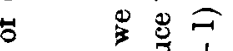

\%

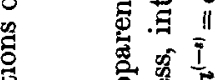

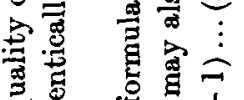

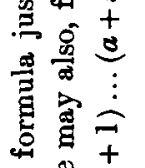

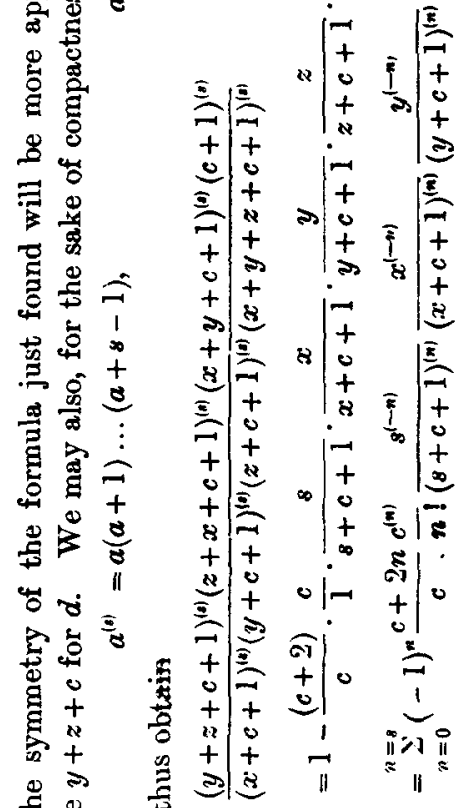

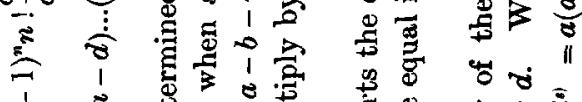

沓

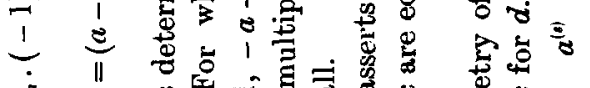

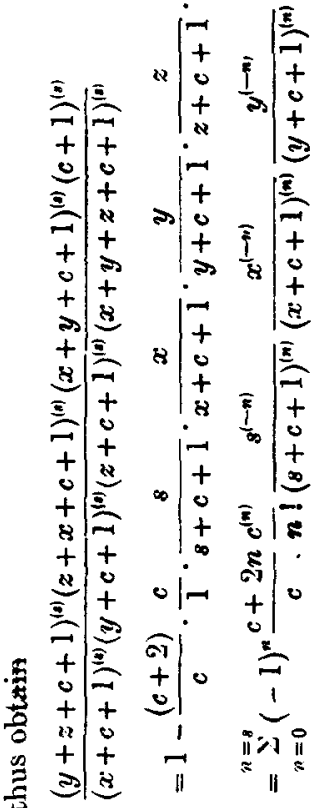

范

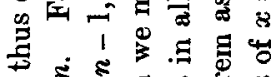

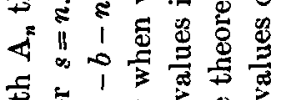

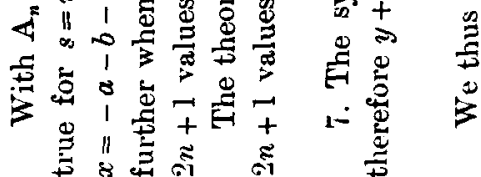

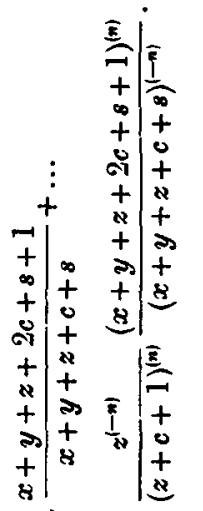

$\stackrel{5}{5}$ 
If we take $c$ as a function of $x, y, z, s$ and a new variable $t$, defined by the equation

so that

$$
x+y+z+2 c+8+1=-t
$$$$
c=-\frac{1}{2}(x+y+z+8+t+1)
$$

then the series on the right of (6) has its general term symmetrical in $x, y, z, 8, t$. It is easy to see that the function on the left is also symmetrical in $x, y, z, t$, for interchange of $x$ and $t$ is equivalent to the substitution of $-x-y-z-2 c-8-1$ for $x$.

It may be pointed out that there is another set of substitutions under which the function on the left of (6) is invariant; $e, g$., $-y,-z, y+z+c$ for $y, z, c$ respectively. If the value (7) of $c$ is first substituted, this is equivalent to change of sign of $y, z$ only. The signs of $x, t$ may also afterwards be changed; the result is the same as that found by writing $-x,-y,-z,-c-8-1$ for $x, y, z, c$ in the series (6). We have thus altogether three different types of expansion of the function on the left of (6).

If we take $x+y+z+c=0, s$ disappears from the general term of the series (6), and the function on the left gives the sum of $s+1$ terms of the series, as may easily be verified by the method of differences.

8. The formula (6), involving five variables, contains, of course, a large number of special or limiting cases. For example, by writing $u-z$ for $c$, and making $z$ infinite, we deduce (2), which itself, when $\beta$ is made infinite, gives (1).

But the most interesting results are those obtained by making the positive integer $s$ infinite, either with or without previous change of variables.

First take (6) as it stands.

Substituting their asymptotic values for the factorials $(\$ 4)$, we find for the limit of the function on the left

$$
\frac{\Pi(x+c) \Pi(y+c) \Pi(z+c) \Pi(x+y+z+c)}{\bar{\Pi}(y+z+c) \Pi(z+x+c) \Pi(x+y+c) \Pi c} .
$$

As for the series, we shall show that its limit can be found term by term, as in the case of the series (2), subject to a certain restriction on the values of the variables. 
The series of term by term limits is

$$
\sum_{n=0}^{n=\infty}(-1)^{n} \frac{c+2 n}{c} \frac{c^{(n)}}{n !} \frac{x^{(-n)}}{(x+c+1)^{(n)}} \frac{y^{(-n)}}{(y+c+1)^{(n)}} \frac{z^{(-n)}}{(z+c+1)^{(n)}},
$$

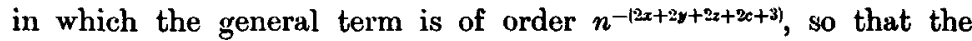
series converges absolutely if $\mathrm{R}(x+y+z+c)>-1$.

But if we write $\sigma$ for $x+y+z+c$, the factor containing $s$ in the general term of $(6)$ is

$$
\frac{s \ldots \ldots(s-n+1)}{(s+c+1) \ldots(s+c+n)} \cdot \frac{(s+\sigma+c+1) \ldots(s+\sigma+c+n)}{(s+\sigma) \ldots(s+\sigma-n+1)} .
$$

This is got from the corresponding factor of the previous term by multiplying by

$$
\frac{(s-n+1)(s+\sigma+c+n)}{(s+c+n)(s+\sigma-n+1)} \text { or } \frac{1+\frac{\sigma}{s+c+n}}{1+\frac{\sigma}{s-n+1}},
$$

which, supposing for brevity that $c$ and $\sigma$ are real, is not greater than $l$ if $\sigma$ is not negative and

$$
8+c+n>8-n+1 \text {, that is, } n>\frac{1}{2}(1-c) \text {. }
$$

Under these conditions, if $\mathbf{N}$ be any positive integer greater than $\frac{1}{2}(1-c)$, the value of the factor $(8)$ for any $n$ greater than $N$ cannot exceed its value for $n=N$, which has obviously a finite upper limit independent of 8 .

The residue after $n$ terms in (6) cannot therefore exceed a certain finite multiple of the corresponding residue in the series of limits. Hence, as in $\$ 4$, we can take the limit of the series in (6) term by term, and obtain

$$
\begin{gathered}
\frac{\Pi(x+c) \Pi(y+c) \Pi(z+c) \Pi(x+y+z+c)}{\Pi(y+z+c) \Pi(z+x+c) \Pi(x+y+c) \Pi c} \\
=\sum_{n=0}^{n=\infty}(-1)^{n} \frac{c+2 n}{c} \frac{c^{(n)}}{n !} \frac{x^{(-n)}}{(x+c+1)^{(n)}} \frac{y^{(-n)}}{(y+c+1)^{(n)}} \frac{z^{(-n)}}{(z+c+1)^{(\boldsymbol{n})}} .
\end{gathered}
$$

The theorem has been proved for $x+y+z$ and $c$ real, and $x+y+z+c>-1$. It is, in fact, true if only $R(x+y+z+c)>-1$.

For the extension we may be content to rely on the general Theorem of Continuation in the Theory of Functions. 


\section{3}

9. Suppose we consider the function expanded in (9) as a function of the complex variable $x$. The infinities of the function are those of $\Pi(x+c)$ and $\Pi(x+y+z+c)$. The series represents the function over part of the $x$ plane, and in this part the first set of infinities, those of $\Pi(x+c)$, are indicated by individual terms becoming infinite. It is worth remarking, as the circumstances often occur, that by a simple change of variable, we may obtain an expansion indicating the other set of infinities. We have only to write $-y,-z, c+y+z$ for $y, z, c$ respectively.

For another instance of the point, in the ordinary hypergeometric series

$$
1+\frac{a \cdot \beta}{1 \cdot \gamma}+\frac{a \cdot a+1 \cdot \beta \cdot \beta+1}{1 \cdot 2 \cdot \gamma \cdot \gamma+1}+\ldots=\frac{\Pi(\gamma-1) \Pi(\gamma-\alpha-\beta-1)}{\Pi(\gamma-a-1) \Pi(\gamma-\beta-1)},
$$

change $\alpha, \beta, \gamma$ into $-\alpha,-\beta, \gamma-\alpha-\beta$ respectively.

10. Some special cases of (9) may be set down.

In (9) put $c=0$. Then

$$
\begin{gathered}
\frac{\Pi x \Pi y \Pi z \Pi(x+y+z)}{\Pi(y+z) \Pi(z+x) \Pi(x+y)} \\
=1-2 \frac{x}{x+1} \cdot \frac{y}{y+1} \cdot \frac{z}{z+1}+2 \frac{x \cdot x-1}{x+1 \cdot x+2} \cdot \frac{y \cdot y-1}{y+1 \cdot y+2} \cdot \frac{z \cdot z-1}{z+1 \cdot z+2}-\ldots, \\
\mathrm{R}(x+y+z)>-1 .
\end{gathered}
$$

In (10) let $\approx$ go to infinity through real positive values. Thus

$$
\begin{array}{r}
\frac{\Pi x \Pi y}{\Pi(x+y)}=1-2 \frac{x}{x+1} \cdot \frac{y}{y+1}+2 \frac{x \cdot x-1}{x+1 \cdot x+2} \cdot \frac{y \cdot y-1}{y+1 \cdot y+2}-\ldots, \\
\mathbf{R}(x+y) \nless-\frac{1}{2} . \quad-\quad-\quad ~-~
\end{array}
$$

In (10) take $z=-\frac{1}{2}$, and get

$$
\begin{gathered}
\Pi x \Pi y \Pi\left(-\frac{1}{2}\right) \Pi\left(x+y-\frac{1}{2}\right) \\
\overline{\Pi\left(x-\frac{1}{2}\right) \Pi\left(y-\frac{1}{2}\right) \Pi(x+y)} \\
=1+2 \frac{x}{x+1} \cdot \frac{y}{y+1}+2 \frac{x \cdot x-1}{x+1 \cdot x+2} \cdot \frac{y \cdot y-1}{y+1 \cdot y+2}+\ldots, \\
\mathrm{R}(x+y)>-\frac{1}{2} . \quad\left(\Pi\left(-\frac{1}{2}\right)=\sqrt{ } \pi\right) .
\end{gathered}
$$

In (12) put $y=+\infty$. Thus

$$
\begin{gathered}
\frac{\Pi x \Pi\left(-\frac{1}{2}\right)}{\Pi\left(x-\frac{1}{2}\right)}=1+2 \frac{x}{x+1}+2 \frac{x \cdot x-1}{x+1 . x+2}+\ldots, \\
R(x) \nless 0 .
\end{gathered}
$$




\section{4}

Interesting results are obtained by making each of the variables half an odd integer in the last four formulæ. For instance, $x=\frac{1}{2}$ in (13) gives Gregory's series for $\pi$.

11. In (9) put $z=-\frac{1}{2} c$. Thus

$$
\begin{gathered}
\sum_{n=0}^{n=\infty} \frac{c^{(n)}}{n !} \frac{x^{(-n)}}{(x+c+1)^{(n)}} \frac{y^{(-n)}}{(y+c+1)^{(n)}} \\
=\frac{\Pi(x+c) \Pi(y+c) \Pi \frac{c}{2} \Pi\left(x+y+\frac{c}{2}\right)}{\Pi\left(x+\frac{c}{2}\right) \Pi\left(y+\frac{c}{2}\right) \Pi(x+y+c) \Pi c} \\
\mathbf{R}\left(x+y+\frac{c}{2}\right)>-1 .
\end{gathered}
$$

In (14) put $x=y=-c$. Hence

$$
\begin{aligned}
\underbrace{n=\infty}_{n=0}\left\{\frac{c(c+1) \ldots(c+n-1)}{n !}\right\}^{3} & =\frac{\Pi \frac{c}{2} \Pi\left(-\frac{3 c}{2}\right)}{\Pi\left(\frac{c}{2}\right) \Pi\left(-\frac{c}{2}\right) \Pi(-c) \Pi c} \\
& =\cos \frac{\pi c}{2} \frac{\Pi\left(-\frac{3 c}{2}\right)}{\left\{\Pi\left(-\frac{c}{2}\right)\right\}^{3}}, \\
(\text { since } \Pi a \Pi(-a) & \left.=\frac{\pi a}{\sin \pi \alpha}\right) ; \quad R(c)<\frac{2}{3} .
\end{aligned}
$$

This gives the sum of the cubes of the coefficients of the expansion of $(1-x)^{-c}$. For a proof by means of the theory of hypergeometric functions see Dixon, "Summation of certuin Series," Proc. Lond. Math. Soc., Vol. 35, page 284.

In (9) make $z$ infinite, $x$ and $y=-c$.

Then

$$
\sum_{n=0}^{n=\infty}(-1)^{n} \frac{c+2 n}{c}\left\{\frac{c(c+1) \ldots(c+n-1)}{n !}\right\}^{3}=\frac{\sin \pi c}{\pi c},
$$

In (9) make $x=y=z=-c$. Then

$$
\sum_{n=0}^{n=\infty} \frac{c+2 n}{c}\left\{\frac{c(c+1) \ldots(c+n-1)}{n !}\right\}_{c<\frac{1}{2} .}^{4}=\frac{\sin \pi c}{\pi c} \frac{\Pi(-2 c)}{\{\Pi(-c)\}^{2}} \text {. }
$$


In (9) make $x=y=-c$ and $z+(z+c+1)=0$ or $z=-\frac{c+1}{2}$. Then

$$
\begin{aligned}
& \frac{\sin \pi c}{\pi c} \frac{\Pi\left(\frac{c-1}{2}\right) \Pi\left(-\frac{3 c+1}{2}\right)}{\left\{\Pi\left(-\frac{c+1}{2}\right)\right\}^{2}} \\
& \quad=\sum_{n=0}^{n=\infty} \frac{c+2 n}{c}\left(\frac{c \cdot c+1 \ldots c+n-1}{n !}\right)^{3}
\end{aligned}
$$

12. Another limiting case of the fundamental theorem (6) deserves attention, less perhaps for the result itself than for the unusual way in which one has to go to the limit to obtain it. The example illustrates the necessity of caution before taking the limit of a series term by term, even when a definite limit exists, and the series of term by term limits converges.

In (6) put $c=a-s$ and let $s$ increase indefinitely.

Consider first the sum of the series.

We have

$$
\begin{aligned}
(c+1) \ldots(c+s) & =(a-s+1) \ldots a \\
& =(-1)^{z}(s-a-1) \ldots(-a) \\
& =(-1)^{2} \Pi(s-a-1) / \Pi(-a-1) \\
& =(-1)^{t} s^{-a-1} \Pi s / \Pi(-a-1), \text { asymptotically, }
\end{aligned}
$$

and similarly with the other factorials on the left of $(6)$, the limit of which is therefore

$$
\frac{\Pi(-x-a-1) \Pi(-y-a-1) \Pi(-z-a-1) \Pi(-x-y-z-a-1)}{\overline{\Pi(-y-z-a-1) \Pi(-z-x-a-1) \Pi(-x-y-a-1) \Pi(-a-1)} .}
$$

Next take the series itself. This is

$$
\begin{aligned}
& \sum_{n=0}^{n=8}(-1)^{n} \frac{a-8+2 n}{a-8} \frac{(a-8)^{(n)}}{n !} \frac{8^{(-n)}}{(a+1)^{(n)}} \frac{x^{(-n)}}{(x+a-8+1)^{(n)}} \text { do. } y, z, \\
& \frac{(x+y+z+2 a-s+1)^{(x)}}{(x+y+z+a)^{(-x)}}
\end{aligned}
$$

(the contraction do. $y, z$, signifying that two factors have to be put in, the same functions of $y, z$ respectively as the immediately preceding factor is of $x$ ), and the series of term by term limits is

$$
\sum_{n=0}^{n=\infty} \frac{x^{(-\infty)} y^{(-\infty)} z^{(-\infty)}}{n !(a+1)^{(n)}(x+y+z+a)^{(-n)}}, \quad \text { - - }
$$


章

वे क्ष

\& $\$$

엉

苟高

용.

ఫ施

䒘娄

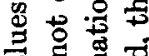

क ज

들 宊

क्षे

는 졃

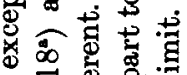

己要

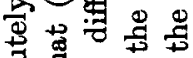

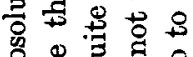

号家完

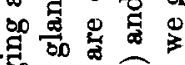

क

高弯

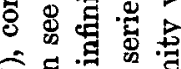

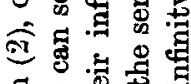

$\Rightarrow$.

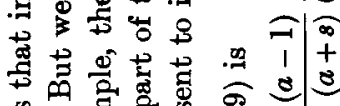

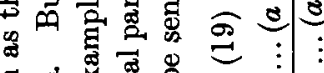

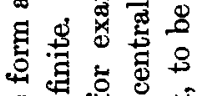

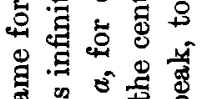

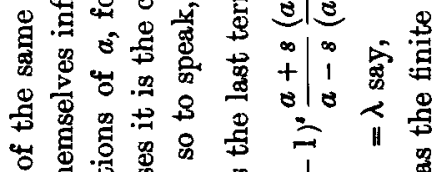

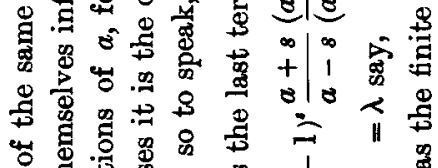

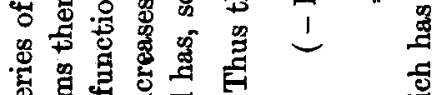

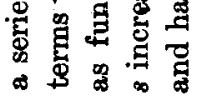

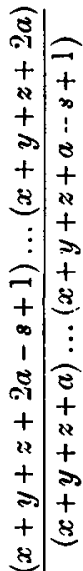

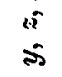

ㅇํㅇ

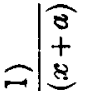

$+$

\&

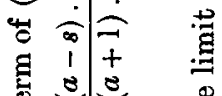

$+\infty \begin{aligned} & \infty \\ & + \\ & 0\end{aligned}$

密
¿

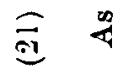

突

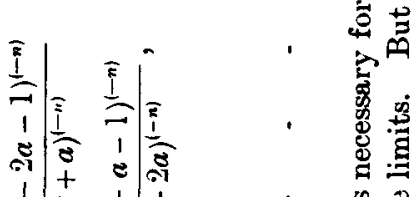

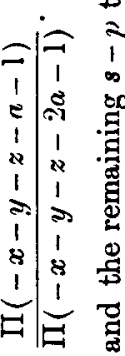

के है

案产

1)

$\begin{array}{ccc}8 & 1 & 0 \\ 1 & 8 & 0 \\ 8 & 1 & 0 \\ 1 & 1 & \end{array}$

当四吉

过 $+\infty$

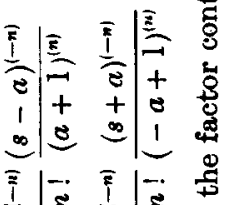

1

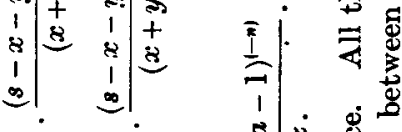

a

के के

용

在

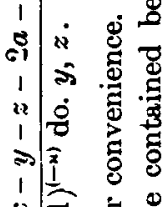

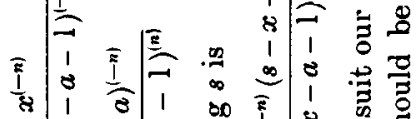

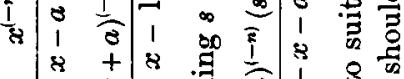

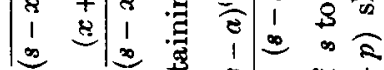

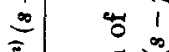

$\frac{\overline{1}}{\infty}$.

i|

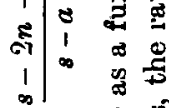

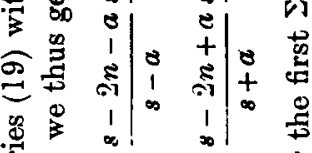

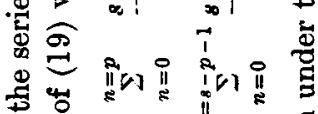

志潀

居

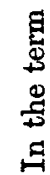

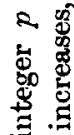

$\stackrel{8}{5}$

运起

. 9

范

3 


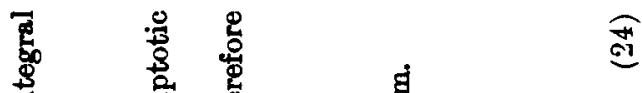

궁

章

总

물

焉

क 帘

意

:

$\vec{i} \quad$ 要

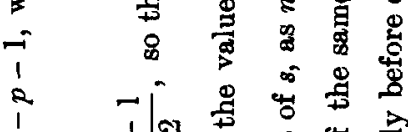

定周度

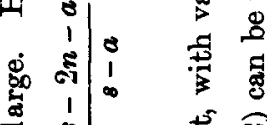

舟

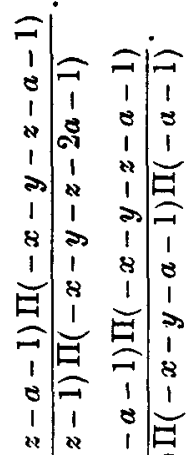

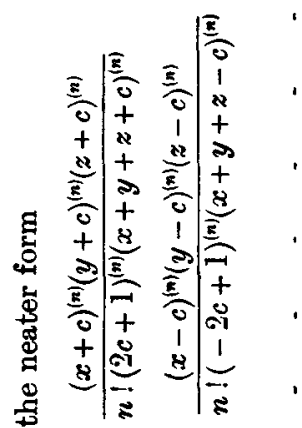

iा

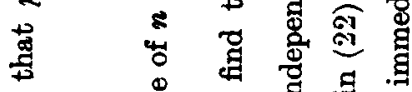

害

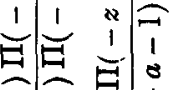

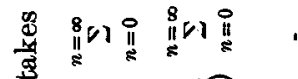

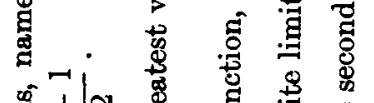

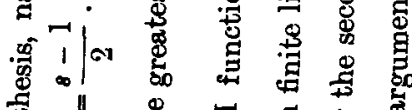

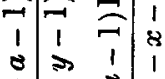

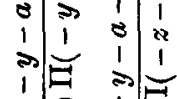

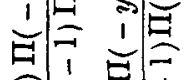

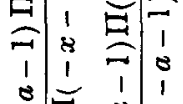

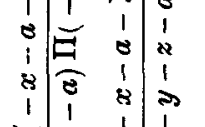

苟苗

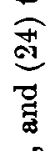

î.

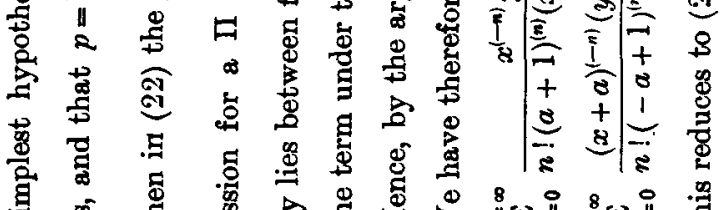

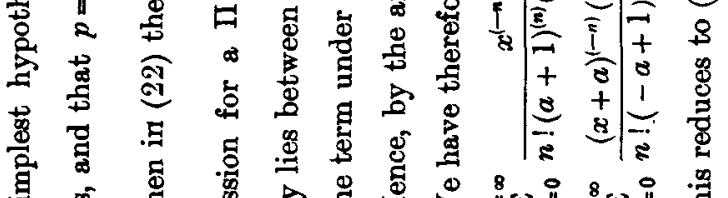

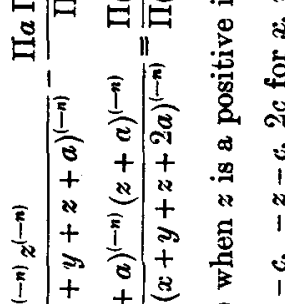

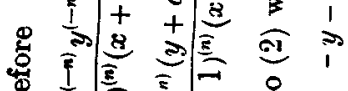

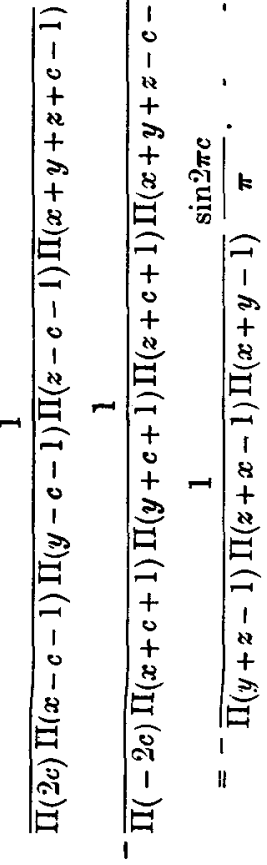

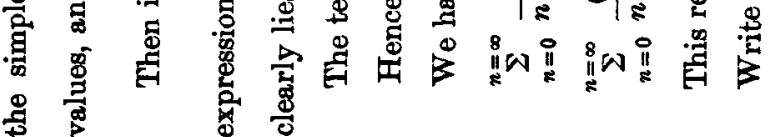


13. For the sake of pointing out some natural extensions of the preceding results, we will now abandon the purely elementary methods hitherto used, and take advantage of the powerful weapon furnished by the Theory of Complex Integration and Residues.

As one of the simplest subjects for the extension contemplated, take the ordinary hypergeometric series

$$
1+\frac{a \beta}{1 \cdot \gamma}+\frac{a \cdot a+1 \cdot \beta \cdot \beta+1}{1 \cdot 2 \cdot \gamma \cdot \gamma+1}+\ldots
$$

This may be written

$$
\frac{\Pi(\gamma-1)}{\Pi(a-1) \Pi(\beta-1)} \sum_{n=0}^{n=\infty} \frac{\Pi(a-1+n) \Pi(\beta-1+n)}{\Pi n \Pi(\gamma-1+n)} .
$$

The sum of the series is known; we proceed to find the sum of the more general series

$$
\mathrm{S}=\sum_{n=-\infty}^{n=\infty} \frac{\Pi(a+n) \Pi(b+n)}{\Pi(c+n) \Pi(d+n)},
$$

which reduces to the preceding when $c$ or $d$ is zero, or an integer.

Consider the function of the complex variable $z$,

$$
f(z)=\frac{\Pi(a+z) \Pi(b+z)}{\Pi(c+z) \Pi(d+z)} \frac{\pi \cos \pi z}{\sin \pi z} .
$$

This function has three sets of simple infinities, namely, those of $\Pi(a+z), \Pi(b+z)$ and $1 / \sin \pi z$; the series $S$ is obviously the sum of the residues of the function at the poles of $1 / \sin \pi z$.

It may be proved that, subject to a restriction on the values of $a, b, c, d$, the sum of the residues of $f(z)$ at all its poles is zero.

To prove this, we have to consider the form of the uniform function $f(z)$ for $z$ infinite.

Now, when $z$ tends to infinity, we have the asymptotic expression

$$
\Pi(z)=\sqrt{2 \pi} e^{(z+1) \log x-z},
$$

valid on the supposition that the argument or phase of $z$ lies between $-\pi$ and $\pi$. If the real part of $z$ is large and negative, but its imaginary part is not large, the form (28) fails; but in this case the asymptotic form of $\Pi(z)$ is easily deduced from the fundamental relation

$$
\Pi z \Pi(-z)=\pi z / \sin \pi z
$$

From (28) it follows easily that, asymptotically,

$$
\Pi(z+a)=z^{a} \Pi(z) \text {. }
$$


Hence, unless the phase of $z$ tends to $\pm \pi$, we have

$$
\frac{\Pi(a+z) \Pi(b+z)}{\Pi(c+z) \Pi(d+z)}=z^{a+b-c-d}, \text { asymptotically. }
$$

In the excepted case, write $z=-\zeta$; then

$$
\begin{aligned}
\Pi(a+z) & =\Pi(a-\zeta) \\
& =\frac{1}{\Pi(\zeta-a)} \frac{\pi(\zeta-a)}{\sin \pi(\zeta-a)},
\end{aligned}
$$

and $\frac{\Pi(a+z) \Pi(b+z)}{\Pi(c+z) \bar{\Pi}(d+z)}=\zeta^{a+b-c-d} \frac{\sin \pi(z+c) \sin \pi(z+d)}{\sin \pi(z+a) \sin \pi(z+b)}$, asymptotically.

It may easily be proved that we can, avoiding any zero of $\sin \pi(z+a)$ or $\sin \pi(z+b)$ or $\sin \pi z$, describe a circle in the $z$ plane of radius $r$ as large as we please, along which

$$
\left|\frac{\sin \pi(z+c) \sin \pi(z+d) \cos \pi z}{\sin \pi(z+a) \sin \pi(z+\bar{b}) \sin \pi z}\right|
$$

has a finite upper limit independent of $r$.

Also the integral $\int\left|z^{n+b-c-d} d z\right|$ taken along the circle will tend to zero as $r$ increases, provided the real part of $a+b-c-d$ is less than -1 .

Hence if $\mathrm{R}(a+b-c-d)<-1$, the sum of the residues of $f(z)$ is zero.

The residue of $\Pi(z+a)$ at the pole $z=-a-p-1(p=0,1, \ldots)$ is $(-1)^{p} / \Pi p$.

The residue of $f(z)$ at this pole is therefore

$$
\frac{(-1)^{p}}{\Pi p} \frac{\Pi(b-a-p-1)}{\Pi(c-a-p-1) \Pi(d-a-p-1)}(-\pi \cot \pi a),
$$

and the sum of the residues at the poles of $\Pi(a+z)$ is

$$
\begin{aligned}
& -\pi \cot \pi a \frac{\Pi(b-a-1)}{\Pi(c-a-1) \Pi(d-a-1)} \\
& \left\{1+\frac{a-c+1 \cdot a-d+1}{1 \cdot a-b+1}+\frac{a-c+1 \cdot a-c+2 \cdot a-d+1 \cdot a-d+2}{1 \cdot 2 \cdot a-b+1 \cdot a-b+2}+\ldots\right\} \\
& =-\pi \cot \pi a \frac{\Pi(b-a-1)}{\Pi(c-a-1) \Pi(d-a-1)} \cdot \frac{\Pi(a-b) \Pi(c+d-a-b-2)}{\Pi(c-b-1) \Pi(d-b-1)} \\
& =\pi^{2} \frac{\cot \pi a}{\sin \pi(a-b)} \frac{\Pi(c+d-a-b-2)}{\Pi(c-a-1) \Pi(d-a-1) \Pi(c-b-1) \Pi(d-b-1)} .
\end{aligned}
$$


For the sum of the residues at the poles of $\mathrm{II}(b+z)$ we have only to interchange $a$ and $b$ in this.

Hence

$\mathrm{S}=-$ sum of residues at poles of $\Pi(z+a)$ and $\Pi(z+b)$

$$
=\frac{\pi^{2}}{\sin \pi a \sin \pi b} \frac{\Pi(c+d-a-b-2)}{\Pi(c-a-1) \Pi(d-a-1) \Pi(c-b-1) \Pi(d-b-1)} .
$$

We may write

$$
\begin{aligned}
\mathrm{S} & =\sum_{n=0}^{n=\infty}+\sum_{n=-1}^{n=-\infty} \\
& =\frac{\Pi a \Pi b b}{\Pi c \bar{\Pi} d}\left(\begin{array}{c}
1+\frac{a+1}{c+1} \cdot \frac{1}{c d} d+1 \\
+\frac{a+1 \cdot a+2 \cdot b+1 \cdot b+2}{c+1 \cdot c+2 \cdot d+1 \cdot d+2}+\ldots \\
+\frac{c d}{a b}+\frac{c \cdot c-1 \cdot d \cdot d-1}{a \cdot a-1 \cdot b \cdot b-1}+\ldots
\end{array}\right)
\end{aligned}
$$

For $a, b$ write $-a-1,-b-1$ and the result becomes

$$
\begin{aligned}
& 1+\frac{a b}{c+1 \cdot d+1}+\frac{a \cdot a-1 \cdot b \cdot b-1}{c+1 \cdot c+2 \cdot d+1 \cdot d+2}+\ldots \\
&+ \frac{c d}{a+1 \cdot b+1}+\frac{c \cdot c-1 \cdot d \cdot d-1}{a+1 \cdot a+2 \cdot b+1 \cdot b+2}+\ldots \\
&=\frac{\Pi a \Pi b \Pi c \Pi d \Pi(a+b+c+d)}{\Pi(a+c) \Pi(a+d) \Pi(b+c) \Pi(b+d)} \cdot \quad-\quad- \\
& \mathrm{R}(a+b+c+d)>-1 .
\end{aligned}
$$

This reduces to (3) when $d$ is zero.

14. The result (9), which reduces to the summation of the hypergeometric series, just generalised, when we put $z=u-c$ and make $c$ infinite, may be extended by a similar process. After putting $x-c, y-c, z-c$ for $x, y, z$ in (9), we may write it in the form

$$
\begin{aligned}
& \sum_{n=0}^{n=\infty}(c+2 n) \frac{\Pi(c-1+n)}{\Pi n} \frac{\Pi(c-1-x+n)}{\Pi(x+n)} \text { do. } y, z \\
& =\frac{\Pi(c-x-1) \Pi(c-y-1) \Pi(c-z-1) \Pi(x+y+z-2 c)}{\Pi(y+z-c) \Pi(z+x-c) \Pi(x+y-c)} .
\end{aligned}
$$

With the help of this theorem and the method of residues, we can sum the more general series

$$
\mathrm{S}=\sum_{n=-\infty}^{n=\infty}(c+2 n) \frac{\Pi(c-1-t+n)}{\Pi(t+n)} \text { do. } x, y, z,
$$

which reduces to the series of $(32)$ when $t=0$. 
Take the uniform function of $\zeta$,

$$
f(\zeta)=(c+2 \zeta) \frac{\Pi(c-1-t+\zeta)}{\Pi(t+\zeta)} \text { do. } x, y, z, \frac{\pi \cos \pi \zeta}{\sin \pi \zeta}
$$

The sum of the residues of the function will be zero, if $\mathrm{R}(t+x+y+z-2 c)>-1$. The sum of the residues at the poles of $1 / \sin \pi \zeta$ is $\mathrm{S}$. For the poles of $\Pi(c-1-t+\zeta)$ the sum is, as in last article,

$$
\begin{gathered}
\pi \cot \pi(t-c) \sum_{n=0}^{n=\infty}(-c+2 t-2 n) \frac{(-1)^{n}}{\Pi n} \frac{1}{\Pi(2 t-c-n)} \frac{\Pi(t-x-n-1)}{\Pi(t+x-c-n)} \text { do. } y, z, \\
=-\cot \pi(t-c) \sin \pi(2 t-c) \frac{\sin \pi(t+x-c)}{\sin \pi(t-x)} \text { do. } y, z, \times \\
\sum_{n=0}^{n=\infty}(c-2 t+2 n) \frac{\Pi(c-1-2 t+n)}{\Pi n} \frac{\Pi(c-1-t-x+n)}{\Pi(x-t+n)} \text { do. } y, z .
\end{gathered}
$$

The sum of the series in the last line is obtained from (32) by writing $c-2 t$ for $c, x-t$ for $x$, etc., and is

$$
\frac{\Pi(c-t-x-1) \Pi(c-t-y-1) \Pi(c-t-z-1) \Pi(t+x+y+z-2 c)}{\Pi(y+z-c) \Pi(z+x-c) \Pi(x+y-c)} .
$$

Hence (34) becomes

$$
\begin{aligned}
& \pi^{s} \frac{\Pi(t+x+y+z-2 c)}{\Pi(y+z-c) \Pi(z+x-c) \Pi(x+y-c) \Pi(t+x-c) \text { do. } y, z} \times \\
& \frac{\sin \pi(2 t-c) \cos \pi(t-c)}{\sin \pi(t-c) \sin \pi(t-x) \text { do. } y, z} \quad-\quad-\quad-\quad-
\end{aligned}
$$

The first line here is symmetrical in $t, x, y, z$; hence the sum of the residues of $f(\zeta)$ at the poles of the four $\Pi$ functions $\Pi(c-1-t+\zeta)$, etc., (that is, $-S$ ), is the product of the first line of (35) by the sum of the four expressions like that in the second line.

To find the sum of these four expressions, consider the function of $\zeta$,

$$
\phi(\zeta)=\frac{\pi \sin \pi(2 \zeta-c) \cos \pi(\zeta-c)}{\sin \pi(\zeta-c) \sin \pi(\zeta-t) \text { do. } x, y, z},
$$

within a strip of the $\zeta$ plane bounded by two lines parallel to the axis of imaginaries and at unit distance apart. On these two lines $\phi(\zeta)$ has equal values, and it vanishes exponential-wise at infinity. By Cauchy's Theorem the sum of the residues of $\phi(\zeta)$ at the poles within the strip accordingly vanishes. 
But the second line of $(35)$ is the residue at the pole of $1 / \sin \pi(\zeta-t)$. Hence the sum of the four similar expressions

$$
\begin{aligned}
& =- \text { residue at pole of } 1 / \sin \pi(\zeta-c) \\
& =-\frac{\sin \pi c}{\sin \pi(t-c) \text { do. } x, y, z} .
\end{aligned}
$$

Thus for the sum of the series in (33) we have

$$
\begin{gathered}
\mathrm{S}=\frac{\pi^{3} \sin \pi c}{\sin \pi(t-c) \text { do. } x, y, z} \times \\
\frac{\Pi(t+x+y+z-2 c)}{\Pi(y+z-c) \Pi(z+x-c) \Pi(x+y-c) \Pi(t+x-c) \text { do. } y, 2}, \\
\text { where } \mathrm{R}(t+x+y+z-2 c)>-1 .
\end{gathered}
$$

To exhibit the result as the summation of a series of rational terms, multiply both sides of (36) by

Then

$$
\frac{\Pi t \Pi x \Pi_{y} \Pi z}{\Pi(c-1-t) \text { do. } x, y, z} .
$$

$$
\begin{aligned}
& c+(c+2) \frac{c-t}{t+1} \text { do. } x, y, z,+\ldots+(c+2 n) \frac{(c-t)^{(n)}}{(t+1)^{(n)}} \text { do. } x, y z,+\ldots \\
& +(c-2) \frac{t}{c-t-1} \text { do. } x, y, z,+\ldots+(c-2 n) \frac{t^{(-n)}}{(c-t-1)^{(-n)}} \text { do. } x, y, z,+\ldots \\
= & \frac{\sin \pi c}{\pi} \frac{\Pi t \Pi x \Pi y \Pi z \Pi(t-c) \Pi(x-c) \Pi(y-c) \Pi(z-c) \Pi(t+x+y+z-2 c)}{\Pi(y+z-c) \Pi(z+x-c) \Pi(x+y-c) \Pi(t+x-c) \Pi(t+y-c) \Pi(t+z-c)} .
\end{aligned}
$$

For $t=0$, this is equivalent to (9).

The result may be put in somewhat more striking form by writing $2 a$ for $c$, and then $t+a, x+a, y+a, z+a$ for $t, x, y, z$.

Of special cases of (37), those obtained by writing $t=c / 2, t=\infty$, $t=(c-1) / 2$ may be mentioned.

On the Resolution of Integral Algebraic Expressions into Factors.

By R. F. Muirhead, M.A., D.Sc.

On Arithmetical Approximations.

By R. F, DAvis, M.A. 